\title{
APPliCATion OF Ultrasonic METHOd FOR MeASURing THE SOUND VELOCITY IN A MEDIUM TO DETERMINE THE TEMPERATURE ChANGE AND FLUCTUATIONS
}

\author{
Christian Halper, Jan Ilko \& Miroslav Rusko
}
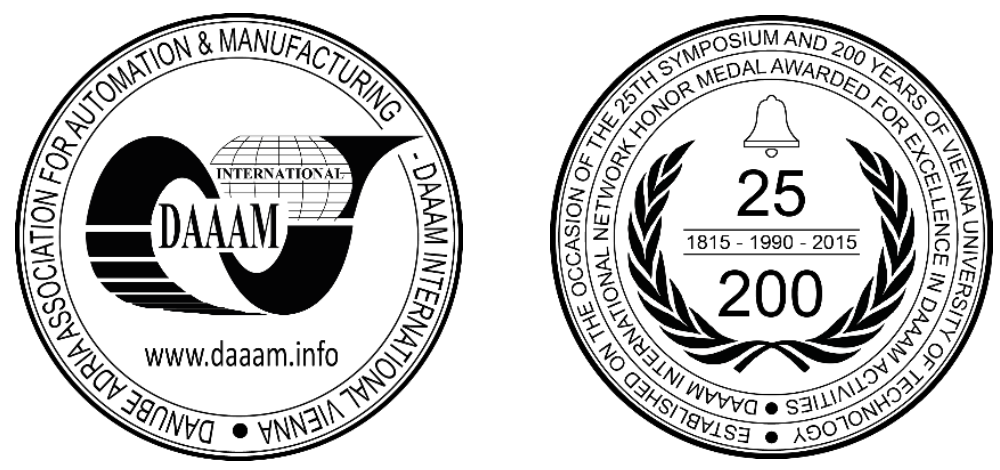

Authors' data: Ing. Halper, C[hristian]*; doc. Ing. PhD. Rusko, M[iroslav]**; Ing. Ilko, J[an]**, *MEDON GmbH, Olbendorf, Austria, **Slovak University of Technology in Bratislava, UIBE, Trnava, Slovakia, christian.halper@medon.at, jan.ilko@stuba.sk, miroslav.rusko@stuba.sk

This Publication has to be referred as: Halper, C[hristian]; Ilko, J[an] \& Rusko, M[iroslav] (2018). Application of Ultrasonic Method for Measuring the Sound Velocity in a Medium to Determine the Temperature Change and Fluctuations, Proceedings of the 29th DAAAM International Symposium, pp.0687-0697, B. Katalinic (Ed.), Published by DAAAM International, ISBN 978-3-902734-20-4, ISSN 1726-9679, Vienna, Austria

DOI: $10.2507 / 29$ th.daaam.proceedings.099

\begin{abstract}
There are application in technology processes where the fast determination of the temperature changes is important to achieve a stable process. The standard temperature sensors has a specific time-period to provide the information about the real temperature of the media. To get a fast response there is a possibility to use an ultrasonic method to determine the change and the real temperature as well. The goal of this study is to test the response time, correlation and reliability of ultrasonic measurement method for determination of water temperature as a part of further drinking water studies and analysis.
\end{abstract}

Keywords: temperature; ultrasonic; measurement; monitoring

\section{Introduction}

The sound velocity of a media is addicted to the media temperature. This fact can be used for temperature change determination or measuring of the temperature as well. For technological applications where the temperature of media is rapidly changing in time is the ultrasonic method a good way to get the fast response for the further controlling of the process. Big advantage of this method is by the fast response also the fact that the ultrasonic monitors allow a measuring from outside - so-called clamp on system. The ultrasonic sensors are able to measure in a range of -30 to $+200{ }^{\circ} \mathrm{C}$. There are solutions how to extend this range from cryo-temperatures up to $400{ }^{\circ} \mathrm{C}$. This is called Wave-Injector.

Such method could find its place also in environmental applications. There are authors who are dealing with similar aspects in frame of their work [5], [6], [7]. 


\section{Theory}

Of the many sensing materials available, piezoelectric sensors offer a number of ad vantages [1]. From the value of the velocity of sound it is possible to compute the temperature [9]. A temperature measuring apparatus, shown on the Fig. 1 , measures the temperature of a medium according to the propagation time of ultrasonic waves propagated for a predetermined distance through the medium. The apparatus has a transmitter 11, 12 for transmitting ultrasonic waves having a fixed frequency at predetermined timing and a receiver 13, 14, 15 for receiving the ultrasonic waves and providing a received signal. A delay time detector 24 detects, in response to the received signal, a delay time between the transmission and reception of the ultrasonic waves and a wave number calculator 21 calculates an integral wave number according to the delay time and a period of the ultrasonic waves [3].

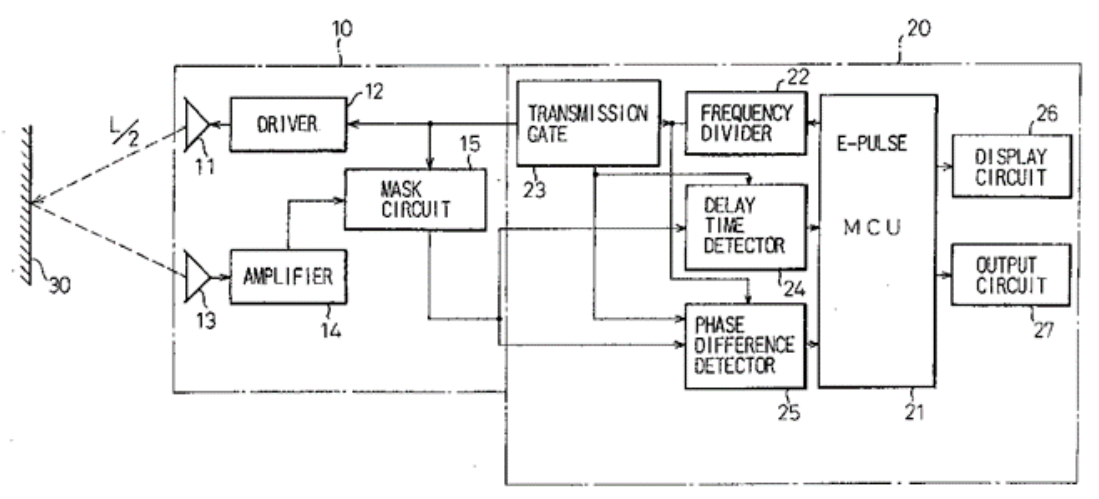

Fig. 1. Ultrasonic monitor principle [3].

For some applications is the response of the fast temperature sensor insufficient either the response is several seconds. Moreover, it si the response of the sensor itself. In real technological applications are the sensors put in a sleave designed for the process where the temperature to be measured. For example there are sensors like LM34 where the real temperature is reached in 3 seconds like shown on the Fig. 2. Producer declare the response in the datasheet. In the Texas Instruments Datasheet for an LM34 (or LM35) in a TO92 package, they give a graph of thermal response in a stirred oil bath [4].

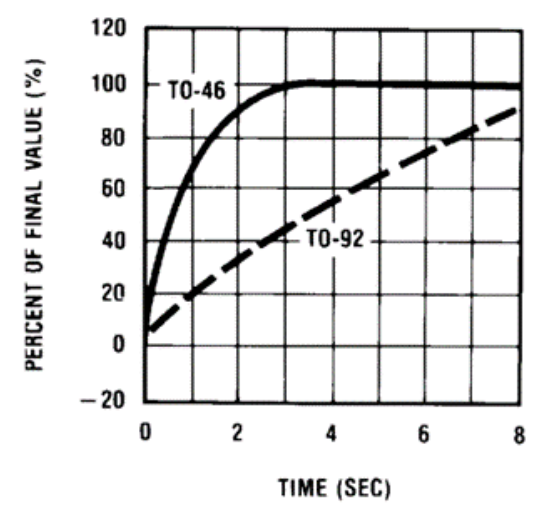

Fig. 2. Thermal Response in Stirred Oil Bath [13].

\section{Dependance of sound speed from the temperature}

The speed of sound in water depends on temperature, salinity and pressure. At a temperature of $25^{\circ} \mathrm{C}$, for example, the speed is $1496 \mathrm{~m} / \mathrm{s}$. In sea water, sound propagates 4.5 times faster than in air. With the increase of any of the factors mentioned (temperature, salinity, pressure), the speed of sound in the water increases. With all the causes, the average speed of sound propagation in fresh water is about $1450 \mathrm{~m} / \mathrm{s}$, and in the sea - about $1500 \mathrm{~m} / \mathrm{s}$ [14]. In all liquids, with the exception of water, the ad. Compressibility with increasing temperature, whereby the density decreases. The speed of sound decreases approximately linearly with increasing temperature. The water occupies a special position in the liquids; here is the ad. Compressibility initially reduced up to a temperature rise to about $60^{\circ} \mathrm{C}$ and only then increases. The speed of sound in water therefore initially has a positive temperature coefficient, which becomes negative when it exceeds 74 ${ }^{\circ} \mathrm{C}$. Thus, the speed increases to a maximum value of $1557 \mathrm{~m} / \mathrm{s}$ at $74{ }^{\circ} \mathrm{C}$. Above this temperature, the speed of sound decreases [8]. 


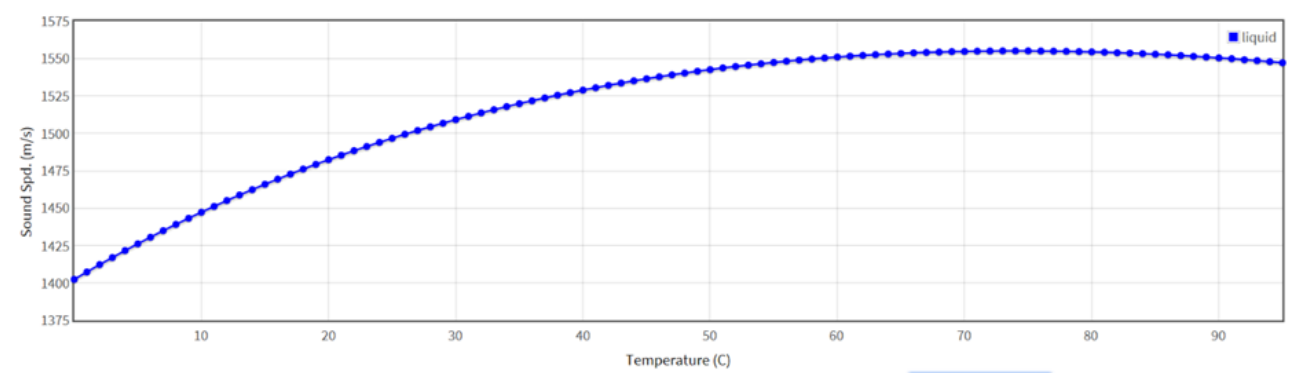

Fig. 3. Function of temperature of water to sound speed at atmospheric pressure [11].

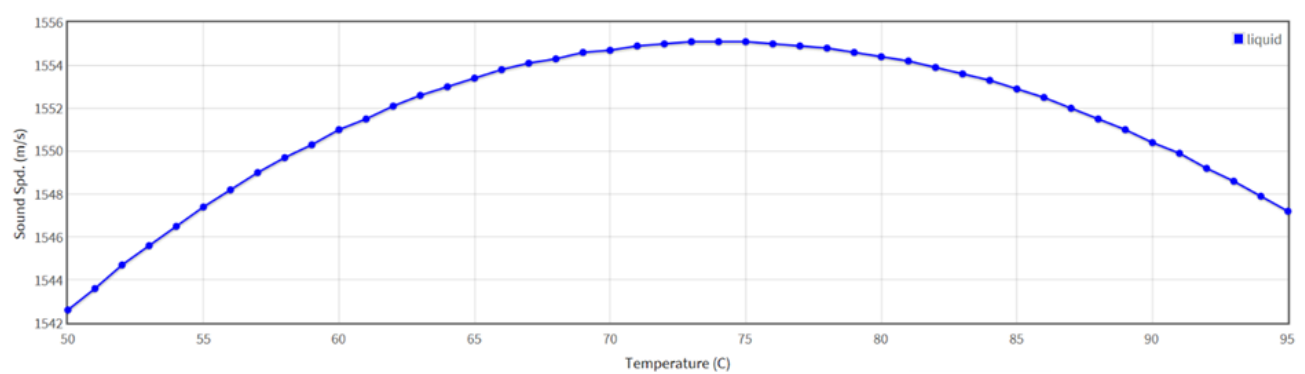

Fig. 4. Function of temperature of water to sound speed at atmospheric pressure in range of $50-95{ }^{\circ} \mathrm{C}[12]$.

\section{Practice}

The goal of the measurement was to see the correlation between the sound speed and the temperature of fluid (water) and the time of response. We used one ultrasonic flow monitor from a german producer with two temperature inputs (Fig. 5). For sound speed measurement has been used ultrasonic sensors with $1 \mathrm{MHz}$ operating frequency. The temperature measument was done with two paired Pt100 sensors (Fig. 6).
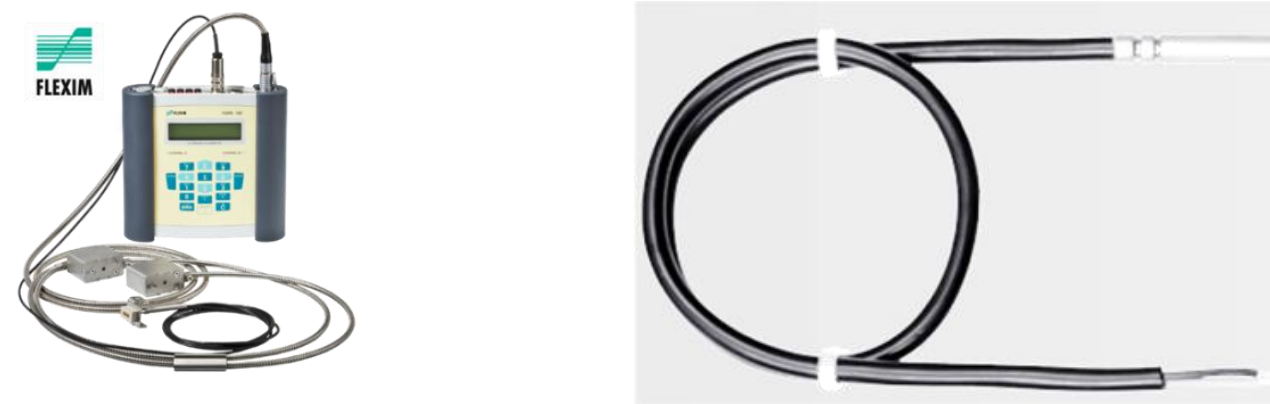

Fig. 5. Ultrasonic flow monitor F601 [2].

Fig. 6. Temperature sensor Pt 100 [10].

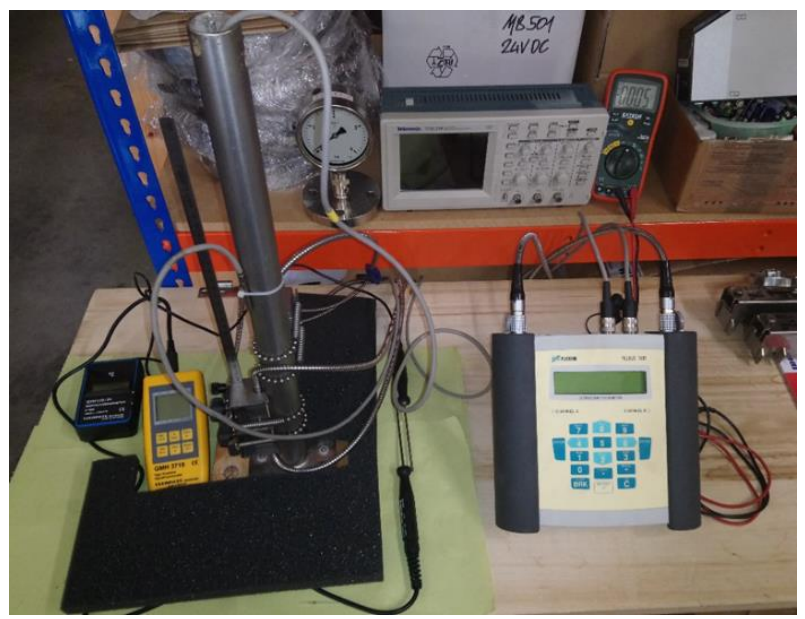

Fig. 7. Measurement assembly. 


\section{Measurement assemblies}

There have been done two measurements.

- The first one was to compare the reaction of temperature sensor insitu with tempoerature sensor exsitu and the sound speed change of the medium. The measurement assembly is shown on the fig. 7. Test 1.

- The second measurement has been performed with two temperature sensors put into the medium and the sound speed monitor. For this measurement was taken a tube with a diameter of $110 \mathrm{~mm}$. Test 2.

Test 1: The first measurement was performed with one temperature senssor (T1) in the media and the second sensor (T2) outside to see the time response on the temperature change, as mentioned above. The setting of the controll unit is shown on the following table (Table 1).

\begin{tabular}{|c|c|c|c|}
\hline \multicolumn{4}{|c|}{ General } \\
\hline Physical quantity & Unit & & \\
\hline Transducer serial no. & & $\mathrm{CDQ1N}$ & 749 \\
\hline \multicolumn{4}{|c|}{ Parameters } \\
\hline Physical quantity & Unit & & \\
\hline Measurement task & & Sound & \\
\hline Function & & & \\
\hline Measuring point & & & \\
\hline Outer diameter & $\mathrm{mm}$ & & \\
\hline Pipe wall material & & Stainle & \\
\hline Pipe wall thickness & $\mathrm{mm}$ & & \\
\hline Pipe wall roughness & $\mathrm{mm}$ & & \\
\hline Roughness & $\mathrm{mm}$ & $0.00(7$ & \\
\hline Fluid type & & & \\
\hline Fluid & & & \\
\hline Fluid temperature & ${ }^{\circ} \mathrm{C}$ & 25.00 & $T 2$ \\
\hline Fluid sound speed & $\mathrm{m} / \mathrm{s}$ & \multicolumn{2}{|c|}{1496.55} \\
\hline Cable length & $\mathrm{m}$ & \multicolumn{2}{|c|}{0} \\
\hline \multicolumn{4}{|c|}{ Measurement } \\
\hline Physical quantity & Unit & \multicolumn{2}{|c|}{ A } \\
\hline Channel activated & & \multicolumn{2}{|c|}{ Yes } \\
\hline Sound paths & & \multicolumn{2}{|c|}{2} \\
\hline Transducer distance & $\mathrm{mm}$ & \multicolumn{2}{|c|}{4.00} \\
\hline
\end{tabular}

Table 1. Flow monitor control unit setup during Test 1.

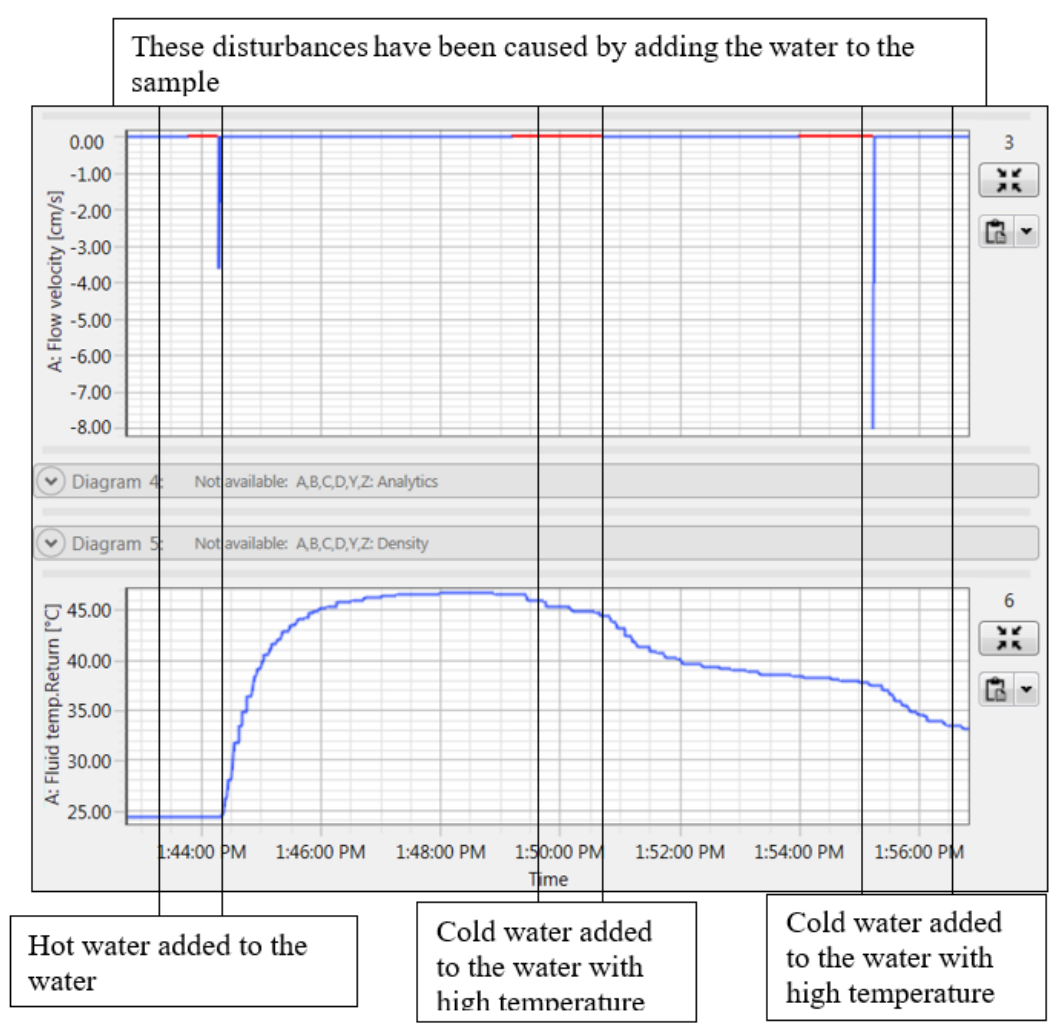

Fig. 8. Process of media temperature changing during the test 1. 
The collected data have been exported to xls - see Table 2.

\begin{tabular}{|c|c|c|c|c|}
\hline TEST 1 & Sound speed $[\mathrm{m} / \mathrm{s}]$ & Aux, temp $\left[{ }^{\circ} \mathrm{C}\right]$ & Fluid temp $\left[{ }^{\circ} \mathrm{C}\right]$ & T-Diff $\left[{ }^{\circ} \mathbf{C}\right]$ \\
\hline 7/17/2018 1:44:20 PM & 1516,7513 & 23,00 & 24,30 & 1,30 \\
\hline 7/17/2018 1:44:30 PM & 1515,6481 & 22,80 & 28,10 & 5,30 \\
\hline 7/17/2018 1:44:40 PM & 1515,0974 & 22,70 & 33,40 & 10,70 \\
\hline 7/17/2018 1:44:50 PM & 1514,5682 & 22,70 & 36,40 & 13,70 \\
\hline 7/17/2018 1:45:00 PM & 1514,2531 & 25,70 & 39,20 & 13,50 \\
\hline 7/17/2018 1:45:10 PM & 1513,8198 & 33,50 & 41,10 & 7,60 \\
\hline 7/17/2018 1:45:20 PM & 1514,0007 & 39,10 & 42,10 & 3,00 \\
\hline 7/17/2018 1:45:30 PM & 1513,4451 & 42,90 & 43,50 & 0,60 \\
\hline 7/17/2018 1:45:40 PM & 1513,5037 & 44,80 & 44,10 & $-0,70$ \\
\hline 7/17/2018 1:45:50 PM & 1513,2167 & 46,90 & 44,70 & $-2,20$ \\
\hline 7/17/2018 1:46:00 PM & 1513,2736 & 48,40 & 45,20 & $-3,20$ \\
\hline 7/17/2018 1:46:10 PM & 1513,3082 & 49,20 & 45,30 & $-3,90$ \\
\hline 7/17/2018 1:46:20 PM & 1512,9294 & 49,80 & 45,80 & $-4,00$ \\
\hline 7/17/2018 1:46:30 PM & 1512,9211 & 50,50 & 45,80 & $-4,70$ \\
\hline 7/17/2018 1:46:40 PM & 1512,9133 & 51,10 & 46,00 & $-5,10$ \\
\hline 7/17/2018 1:46:50 PM & 1512,8199 & 51,10 & 46,30 & $-4,80$ \\
\hline 7/17/2018 1:47:00 PM & 1512,5710 & 51,30 & 46,30 & $-5,00$ \\
\hline 7/17/2018 1:47:10 PM & 1512,6099 & 51,50 & 46,40 & $-5,10$ \\
\hline 7/17/2018 1:47:20 PM & 1512,5538 & 51,50 & 46,50 & $-5,00$ \\
\hline 7/17/2018 1:47:30 PM & 1512,2006 & 51,70 & 46,50 & $-5,20$ \\
\hline 7/17/2018 1:47:40 PM & 1512,1123 & 51,70 & 46,60 & $-5,10$ \\
\hline 7/17/2018 1:47:50 PM & 1511,8711 & 51,70 & 46,60 & $-5,10$ \\
\hline 7/17/2018 1:48:00 PM & 1512,5986 & 51,70 & 46,70 & $-5,00$ \\
\hline 7/17/2018 1:48:10 PM & 1512,0269 & 51,70 & 46,70 & $-5,00$ \\
\hline 7/17/2018 1:48:20 PM & 1511,9932 & 51,60 & 46,70 & $-4,90$ \\
\hline 7/17/2018 1:48:30 PM & 1511,9890 & 51,60 & 46,70 & $-4,90$ \\
\hline 7/17/2018 1:48:40 PM & 1511,8707 & 51,50 & 46,70 & $-4,80$ \\
\hline 7/17/2018 1:48:50 PM & 1512,0460 & 51,40 & 46,70 & $-4,70$ \\
\hline 7/17/2018 1:49:00 PM & 1511,7501 & 50,10 & 46,60 & $-3,50$ \\
\hline 7/17/2018 1:49:10 PM & 1511,1442 & 47,70 & 46,60 & $-1,10$ \\
\hline 7/17/2018 1:50:50 PM & 1504,5839 & 37,10 & 43,90 & 6,80 \\
\hline 7/17/2018 1:51:00 PM & 1505,2888 & 36,60 & 43,10 & 6,50 \\
\hline 7/17/2018 1:51:10 PM & 1505,9424 & 37,40 & 42,40 & 5,00 \\
\hline 7/17/2018 1:51:20 PM & 1505,5277 & 38,50 & 41,40 & 2,90 \\
\hline 7/17/2018 1:51:30 PM & 1505,5288 & 38,50 & 40,80 & 2,30 \\
\hline 7/17/2018 1:51:40 PM & 1505,5920 & 39,00 & 40,70 & 1,70 \\
\hline 7/17/2018 1:51:50 PM & 1504,9001 & 39,50 & 40,20 & 0,70 \\
\hline 7/17/2018 1:52:00 PM & 1505,2567 & 39,50 & 40,10 & 0,60 \\
\hline 7/17/2018 1:52:10 PM & 1504,8490 & 39,60 & 39,70 & 0,10 \\
\hline 7/17/2018 1:52:20 PM & 1504,9227 & 39,70 & 39,60 & $-0,10$ \\
\hline 7/17/2018 1:52:30 PM & 1504,5078 & 39,70 & 39,30 & $-0,40$ \\
\hline 7/17/2018 1:52:40 PM & 1505,1230 & 39,80 & 39,20 & $-0,60$ \\
\hline 7/17/2018 1:52:50 PM & 1504,6708 & 39,90 & 39,10 & $-0,80$ \\
\hline TEST 1 & Sound speed $[\mathrm{m} / \mathrm{s}]$ & Aux, temp $\left[{ }^{\circ} \mathbf{C}\right]$ & Fluid temp $\left[{ }^{\circ} \mathbf{C}\right]$ & T-Diff $\left[{ }^{\circ} \mathrm{C}\right]$ \\
\hline
\end{tabular}




\begin{tabular}{|c|c|c|c|c|}
\hline 7/17/2018 1:53:00 PM & 1504,6887 & 39,90 & 39,00 & $-0,90$ \\
\hline 7/17/2018 1:53:10 PM & 1504,6481 & 39,90 & 38,90 & $-1,00$ \\
\hline 7/17/2018 1:53:20 PM & 1504,3978 & 39,90 & 38,60 & $-1,30$ \\
\hline 7/17/2018 1:53:30 PM & 1504,1068 & 39,90 & 38,60 & $-1,30$ \\
\hline 7/17/2018 1:53:40 PM & 1504,4713 & 39,90 & 38,50 & $-1,40$ \\
\hline 7/17/2018 1:53:50 PM & 1504,1632 & 39,90 & 38,50 & $-1,40$ \\
\hline 7/17/2018 1:54:00 PM & 1504,5262 & 39,80 & 38,40 & $-1,40$ \\
\hline 7/17/2018 1:55:20 PM & 1493,0122 & 34,20 & 37,50 & 3,30 \\
\hline 7/17/2018 1:55:30 PM & 1493,0046 & 34,10 & 36,50 & 2,40 \\
\hline 7/17/2018 1:55:40 PM & 1493,2284 & 33,60 & 35,90 & 2,30 \\
\hline 7/17/2018 1:55:50 PM & 1493,7861 & 33,10 & 34,90 & 1,80 \\
\hline 7/17/2018 1:56:00 PM & 1493,5598 & 33,00 & 34,50 & 1,50 \\
\hline 7/17/2018 1:56:10 PM & 1493,4324 & 32,80 & 34,00 & 1,20 \\
\hline 7/17/2018 1:56:20 PM & 1494,4509 & 32,80 & 34,00 & 1,20 \\
\hline 7/17/2018 1:56:30 PM & 1494,1292 & 32,80 & 33,50 & 0,70 \\
\hline 7/17/2018 1:56:40 PM & 1493,2693 & 32,80 & 33,40 & 0,60 \\
\hline 7/17/2018 1:56:50 PM & 1493,4330 & 32,70 & 33,10 & 0,40 \\
\hline 7/17/2018 1:57:00 PM & 1493,5544 & 32,70 & 33,10 & 0,40 \\
\hline 7/17/2018 1:57:10 PM & 1493,3602 & 32,70 & 33,00 & 0,30 \\
\hline 7/17/2018 1:57:20 PM & 1493,2382 & 32,70 & 32,90 & 0,20 \\
\hline 7/17/2018 1:57:30 PM & 1493,0513 & 32,70 & 32,80 & 0,10 \\
\hline 7/17/2018 1:57:40 PM & 1493,3098 & 32,70 & 32,60 & $-0,10$ \\
\hline 7/17/2018 1:57:50 PM & 1493,3494 & 32,70 & 32,60 & $-0,10$ \\
\hline 7/17/2018 1:58:00 PM & 1493,3732 & 32,70 & 32,50 & $-0,20$ \\
\hline 7/17/2018 1:58:10 PM & 1492,8188 & 32,70 & 32,50 & $-0,20$ \\
\hline 7/17/2018 1:58:20 PM & 1493,0105 & 32,70 & 32,40 & $-0,30$ \\
\hline 7/17/2018 1:58:30 PM & 1492,8381 & 32,70 & 32,40 & $-0,30$ \\
\hline 7/17/2018 1:58:40 PM & 1492,7958 & 32,70 & 32,30 & $-0,40$ \\
\hline 7/17/2018 1:58:50 PM & 1492,5940 & 32,70 & 32,30 & $-0,40$ \\
\hline 7/17/2018 1:59:00 PM & 1493,0725 & 32,70 & 32,20 & $-0,50$ \\
\hline 7/17/2018 1:59:10 PM & 1492,8896 & 32,70 & 32,20 & $-0,50$ \\
\hline 7/17/2018 1:59:20 PM & 1492,7566 & 32,70 & 32,10 & $-0,60$ \\
\hline 7/17/2018 1:59:30 PM & 1492,4844 & 32,70 & 32,10 & $-0,60$ \\
\hline
\end{tabular}

Table 2. Collected data in reduced intervals from 1 second sampling to 10 seconds intervals.

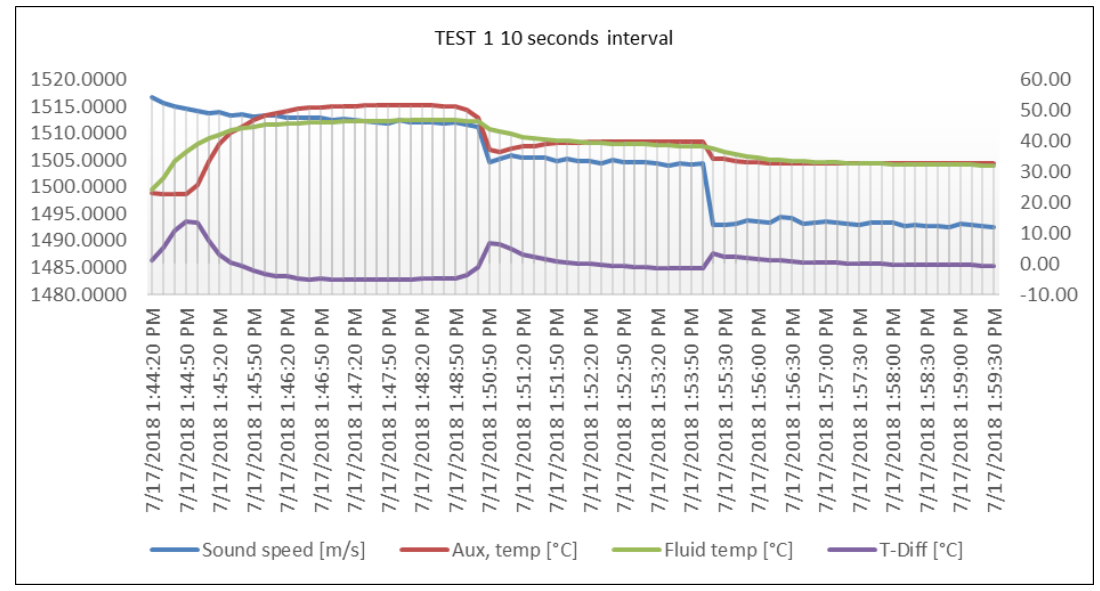

Fig. 9. Trend of the temperatures and sound velocity in Test 1. 
Test 2: The second measurement was performed with both temperature sensors (T1, T2) in the medium. The setting of the control unit is shown on the following table (Table 3).

\begin{tabular}{|c|c|c|c|}
\hline \multicolumn{4}{|c|}{ General } \\
\hline Physical quantity & Unit & \multicolumn{2}{|c|}{$\mathrm{A}$} \\
\hline Transducer serial no. & & \multicolumn{2}{|c|}{ CDM1EZ778779 } \\
\hline \multicolumn{4}{|c|}{ Parameters } \\
\hline Physical quantity & Unit & \multicolumn{2}{|c|}{$\mathrm{A}$} \\
\hline Measurement task & & \multicolumn{2}{|c|}{ Sound speed } \\
\hline Function & & \multicolumn{2}{|c|}{$\mathrm{n} / \mathrm{a}$} \\
\hline Measuring point & & \multicolumn{2}{|c|}{3} \\
\hline Outer diameter & $\mathrm{mm}$ & \multicolumn{2}{|c|}{110.00} \\
\hline Pipe wall material & & \multicolumn{2}{|c|}{ PVC } \\
\hline Pipe wall thickness & $\mathrm{mm}$ & \multicolumn{2}{|c|}{3.40} \\
\hline Pipe wall roughness & $\mathrm{mm}$ & \multicolumn{2}{|c|}{0.00} \\
\hline Roughness & $\mathrm{mm}$ & \multicolumn{2}{|c|}{0.00 (Typical) } \\
\hline Fluid type & & \multicolumn{2}{|c|}{ Liquid } \\
\hline Fluid & & \multicolumn{2}{|c|}{ Water } \\
\hline Fluid temperature & ${ }^{\circ} \mathrm{C}$ & 25.00 & $T 2$ \\
\hline Fluid sound speed & $\mathrm{m} / \mathrm{s}$ & \multicolumn{2}{|c|}{1496.55} \\
\hline Cable length & $\mathrm{m}$ & \multicolumn{2}{|c|}{0} \\
\hline \multicolumn{4}{|c|}{ Measurement } \\
\hline Physical quantity & Unit & \multicolumn{2}{|c|}{$\mathrm{A}$} \\
\hline Channel activated & & \multicolumn{2}{|c|}{ Yes } \\
\hline Sound paths & & \multicolumn{2}{|c|}{2} \\
\hline Transducer distance & $\mathrm{mm}$ & \multicolumn{2}{|c|}{18.50} \\
\hline
\end{tabular}

Table 3. Flow monitor control unit setup during Test 2.

For the second test was taken a PVC tube with diameter of $110 \mathrm{~mm}$.

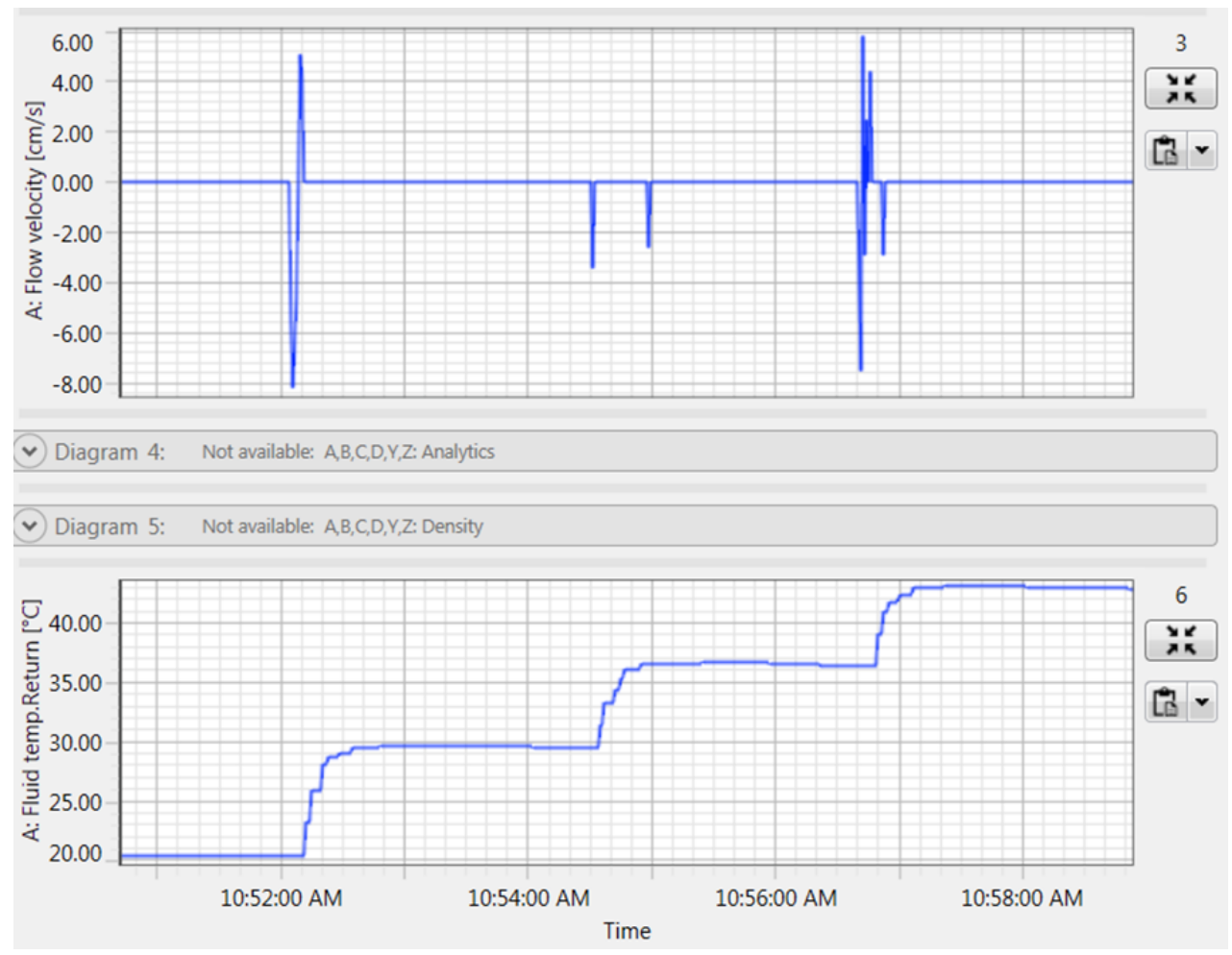

Fig. 10. Process of media temperature changing during the test 2.

The collected data have been exported to xls - see Table 4 . 
29TH DAAAM INTERNATIONAL SYMPOSIUM ON INTELLIGENT MANUFACTURING AND AUTOMATION

\begin{tabular}{|c|c|c|c|c|c|}
\hline TEST 2 & Sound speed $[\mathrm{m} / \mathrm{s}]$ & $\mathbf{T}_{\text {avg }}\left[{ }^{\circ} \mathbf{C}\right]$ & $\mathbf{T} 1\left[{ }^{\circ} \mathbf{C}\right]$ & $\mathbf{T} 2\left[{ }^{\circ} \mathbf{C}\right]$ & $\begin{array}{c}\text { T-Diff } \\
{\left[{ }^{\circ} \mathbf{C}\right]}\end{array}$ \\
\hline 7/18/2018 10:50:50 AM & 1480,36 & 20,20 & 20,10 & 20,30 & 0,20 \\
\hline 7/18/2018 10:51:00 AM & 1480,39 & 20,20 & 20,10 & 20,30 & 0,20 \\
\hline 7/18/2018 10:51:10 AM & 1480,45 & 20,25 & 20,20 & 20,30 & 0,10 \\
\hline $7 / 18 / 2018$ 10:51:20 AM & 1480,35 & 20,25 & 20,20 & 20,30 & 0,10 \\
\hline 7/18/2018 10:51:30 AM & 1480,41 & 20,25 & 20,20 & 20,30 & 0,10 \\
\hline 7/18/2018 10:51:40 AM & 1480,42 & 20,25 & 20,20 & 20,30 & 0,10 \\
\hline $7 / 18 / 2018$ 10:51:50 AM & 1480,33 & 20,25 & 20,20 & 20,30 & 0,10 \\
\hline 7/18/2018 10:52:00 AM & 1480,51 & 20,25 & 20,20 & 20,30 & 0,10 \\
\hline 7/18/2018 10:52:10 AM & 1499,45 & 21,25 & 22,20 & 20,30 & $-1,90$ \\
\hline 7/18/2018 10:52:20 AM & 1506,65 & 27,85 & 27,60 & 28,10 & 0,50 \\
\hline 7/18/2018 10:52:30 AM & 1505,16 & 29,05 & 29,10 & 29,00 & $-0,10$ \\
\hline 7/18/2018 10:52:40 AM & 1505,28 & 29,50 & 29,50 & 29,50 & 0,00 \\
\hline 7/18/2018 10:52:50 AM & 1504,89 & 29,60 & 29,60 & 29,60 & 0,00 \\
\hline 7/18/2018 10:53:00 AM & 1505,26 & 29,65 & 29,70 & 29,60 & $-0,10$ \\
\hline 7/18/2018 10:53:10 AM & 1505,11 & 29,70 & 29,70 & 29,70 & 0,00 \\
\hline 7/18/2018 10:53:20 AM & 1504,80 & 29,70 & 29,70 & 29,70 & 0,00 \\
\hline 7/18/2018 10:53:30 AM & 1504,88 & 29,65 & 29,70 & 29,60 & $-0,10$ \\
\hline 7/18/2018 10:53:40 AM & 1505,19 & 29,65 & 29,70 & 29,60 & $-0,10$ \\
\hline 7/18/2018 10:53:50 AM & 1504,75 & 29,65 & 29,70 & 29,60 & $-0,10$ \\
\hline 7/18/2018 10:54:00 AM & 1504,98 & 29,65 & 29,70 & 29,60 & $-0,10$ \\
\hline 7/18/2018 10:54:10 AM & 1504,83 & 29,60 & 29,70 & 29,50 & $-0,20$ \\
\hline 7/18/2018 10:54:20 AM & 1504,77 & 29,60 & 29,70 & 29,50 & $-0,20$ \\
\hline 7/18/2018 10:54:30 AM & 1514,80 & 29,60 & 29,70 & 29,50 & $-0,20$ \\
\hline 7/18/2018 10:54:40 AM & 1520,34 & 35,30 & 37,30 & 33,30 & $-4,00$ \\
\hline 7/18/2018 10:54:50 AM & 1520,34 & 36,70 & 37,30 & 36,10 & $-1,20$ \\
\hline 7/18/2018 10:55:00 AM & 1519,13 & 36,85 & 37,20 & 36,50 & $-0,70$ \\
\hline 7/18/2018 10:55:10 AM & 1518,93 & 36,70 & 36,80 & 36,60 & $-0,20$ \\
\hline 7/18/2018 10:55:20 AM & 1519,00 & 36,70 & 36,80 & 36,60 & $-0,20$ \\
\hline 7/18/2018 10:55:30 AM & 1518,90 & 36,75 & 36,80 & 36,70 & $-0,10$ \\
\hline 7/18/2018 10:55:40 AM & 1518,97 & 36,75 & 36,80 & 36,70 & $-0,10$ \\
\hline 7/18/2018 10:55:50 AM & 1518,72 & 36,80 & 36,90 & 36,70 & $-0,20$ \\
\hline 7/18/2018 10:56:00 AM & 1518,61 & 36,75 & 36,90 & 36,60 & $-0,30$ \\
\hline 7/18/2018 10:56:10 AM & 1518,43 & 36,70 & 36,80 & 36,60 & $-0,20$ \\
\hline 7/18/2018 10:56:20 AM & 1518,40 & 36,65 & 36,80 & 36,50 & $-0,30$ \\
\hline 7/18/2018 10:56:30 AM & 1518,26 & 36,55 & 36,70 & 36,40 & $-0,30$ \\
\hline 7/18/2018 10:56:40 AM & 1518,07 & 36,55 & 36,70 & 36,40 & $-0,30$ \\
\hline 7/18/2018 10:56:50 AM & 1531,35 & 40,85 & 42,70 & 39,00 & $-3,70$ \\
\hline 7/18/2018 10:57:00 AM & 1529,66 & 43,20 & 44,10 & 42,30 & $-1,80$ \\
\hline 7/18/2018 10:57:10 AM & 1529,18 & 43,60 & 44,20 & 43,00 & $-1,20$ \\
\hline 7/18/2018 10:57:20 AM & 1528,61 & 43,30 & 43,60 & 43,00 & $-0,60$ \\
\hline 7/18/2018 10:57:30 AM & 1528,45 & 43,30 & 43,50 & 43,10 & $-0,40$ \\
\hline 7/18/2018 10:57:40 AM & 1528,36 & 43,20 & 43,30 & 43,10 & $-0,20$ \\
\hline 7/18/2018 10:57:50 AM & 1528,54 & 43,10 & 43,10 & 43,10 & 0,00 \\
\hline 7/18/2018 10:58:00 AM & 1528,24 & 43,10 & 43,10 & 43,10 & 0,00 \\
\hline 7/18/2018 10:58:10 AM & 1528,08 & 43,00 & 43,00 & 43,00 & 0,00 \\
\hline
\end{tabular}




\begin{tabular}{|l|l|l|l|l|l|}
\hline 7/18/2018 10:58:20 AM & 1528,13 & 43,00 & 43,00 & 43,00 & 0,00 \\
\hline 7/18/2018 10:58:30 AM & 1528,18 & 42,95 & 43,00 & 42,90 & $-0,10$ \\
\hline 7/18/2018 10:58:40 AM & 1527,73 & 42,90 & 42,90 & 42,90 & 0,00 \\
\hline $7 / 18 / 2018$ 10:58:50 AM & 1528,03 & 42,90 & 42,90 & 42,90 & 0,00 \\
\hline
\end{tabular}

Table 4. Collected data in reduced intervals from 1 second sampling to 10 seconds intervals.

For the analysis and the conclusion, the data from specified intervals has been taken. The data are in the Table 5 and Fig. $11,12,13$.

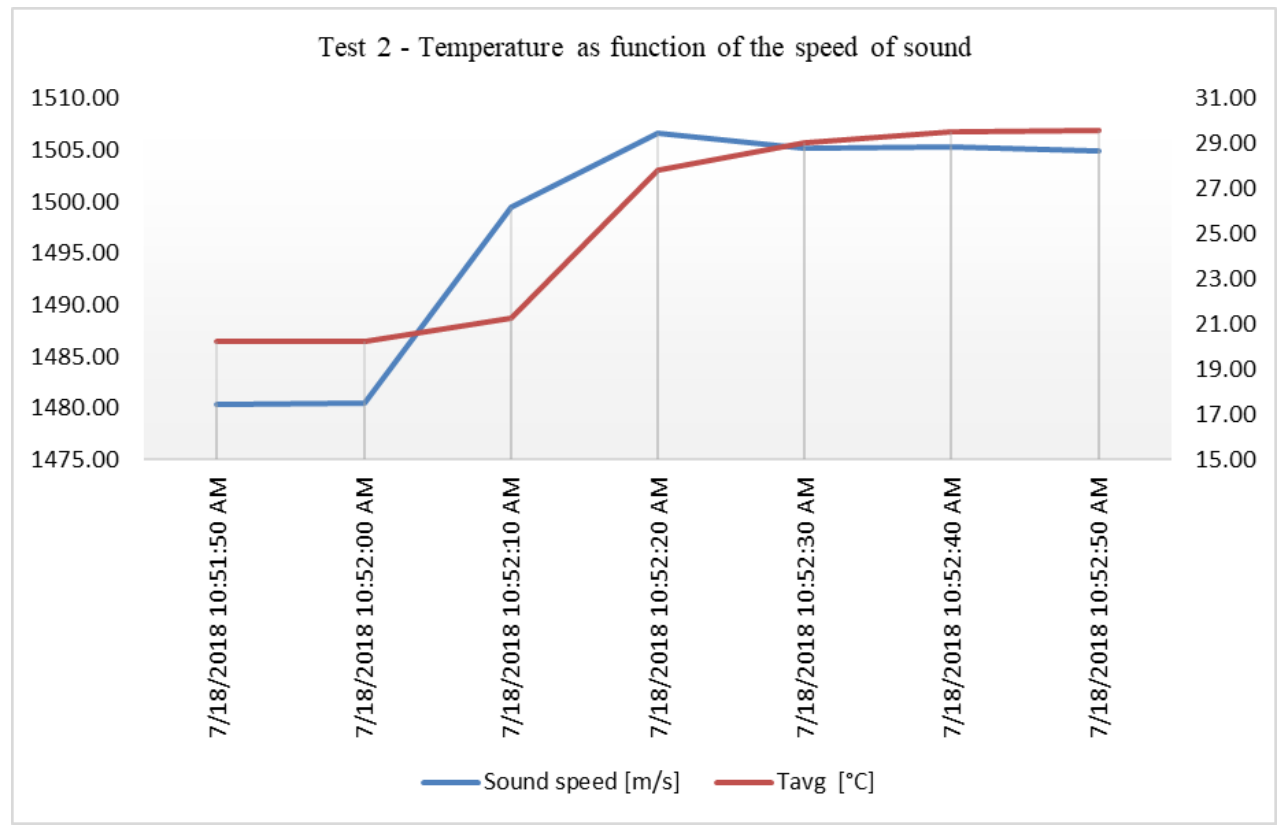

Fig. 11. Trend of the temperatures and sound velocity in Test 2.

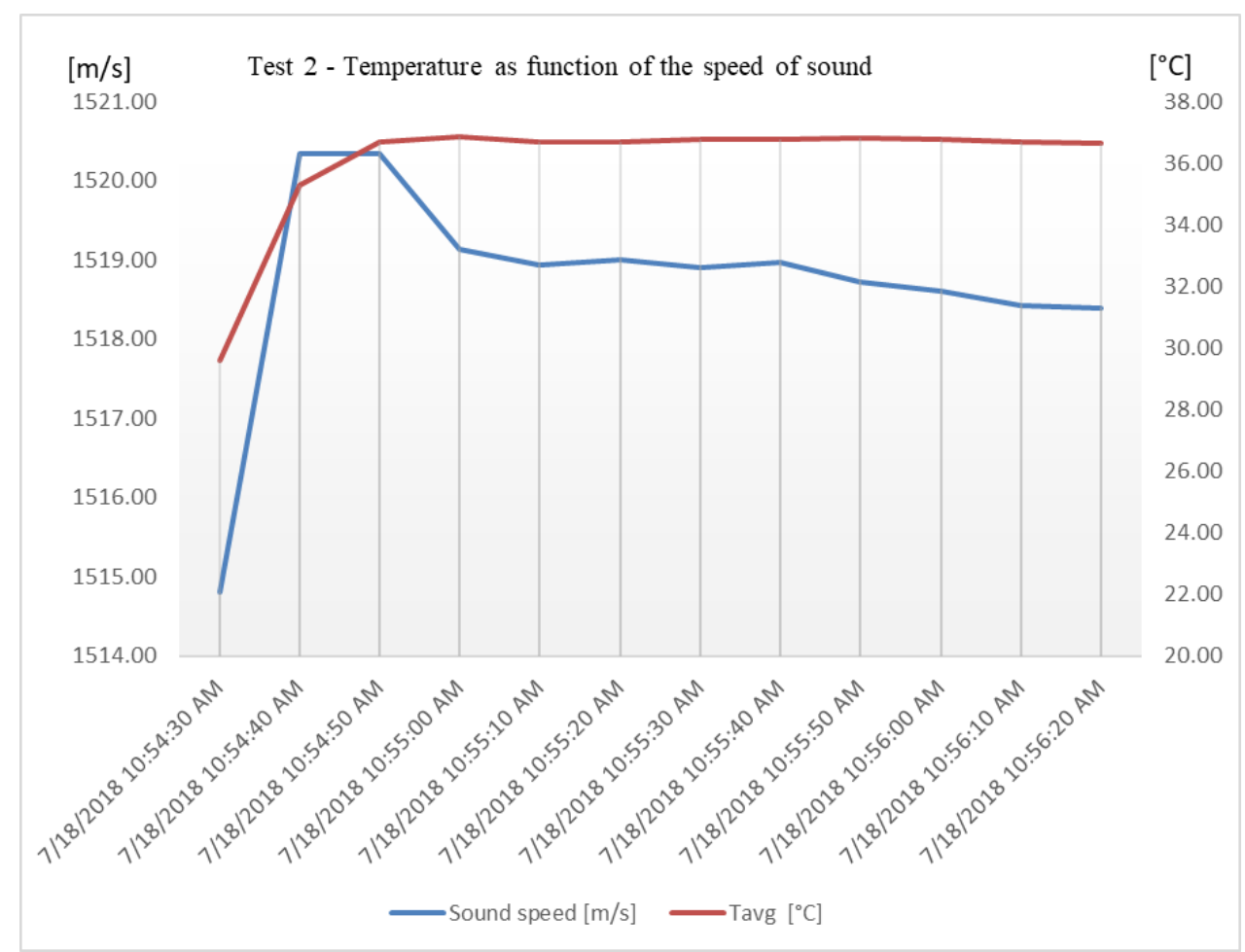

Fig. 12. Trend of the temperatures and sound velocity in Test 2. 


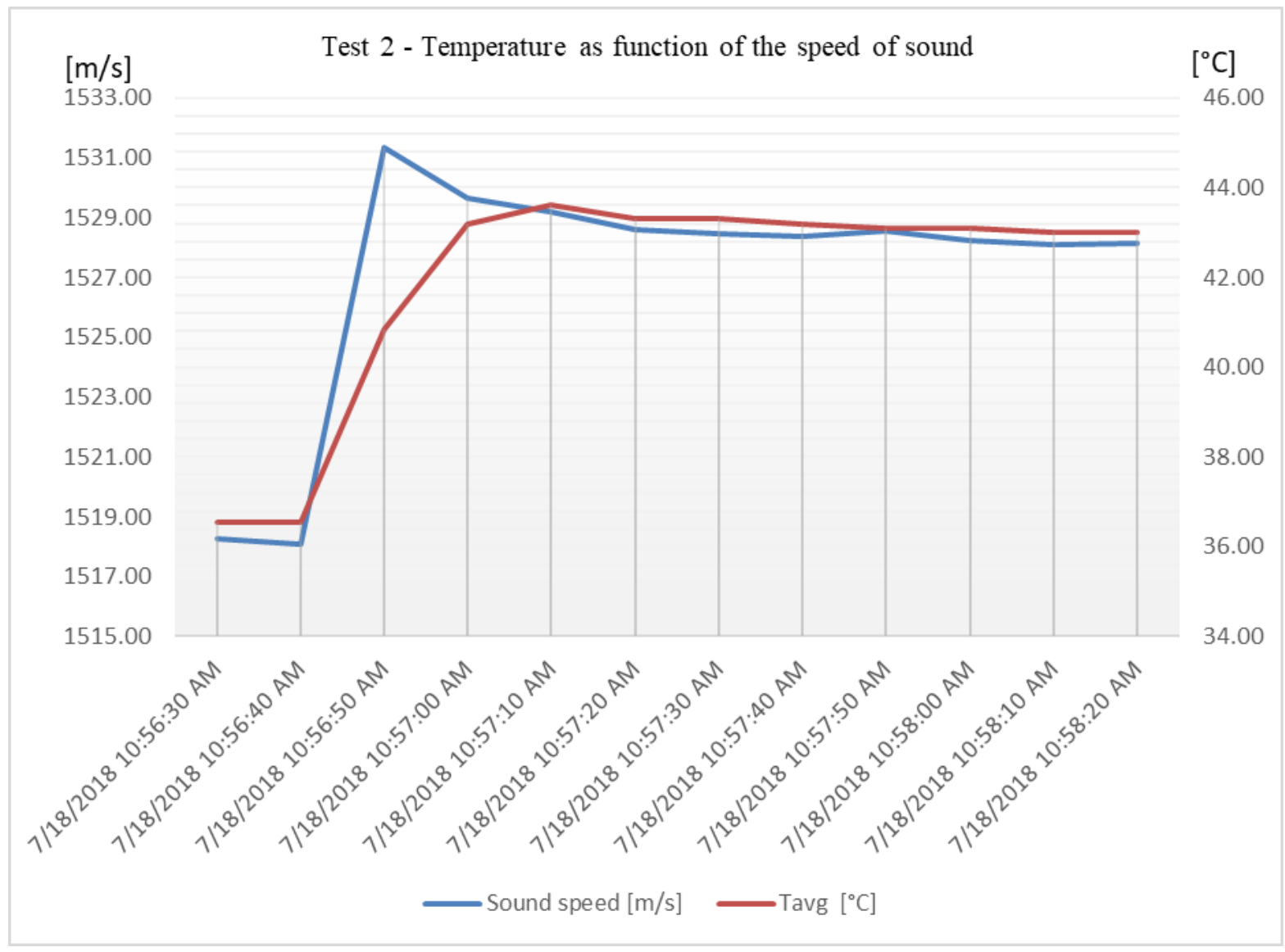

Fig. 13. Trend of the temperatures and sound velocity in Test 2.

\section{Conclusion}

The purpose of the test was to apply a method to monitor the change of drinking water temperature in accuracy of 1 ${ }^{\circ} \mathrm{C}$ due to the risk of increasing the number of bacteria in drinking water at Celsius degree temperature increase. With ultrasound devices the desired accuracy can be achieved. The sound velocity depends on the pressure of the monitored media. The media pressure should be simultaneously monitored either it has a minor effect on the final result. Water temperature monitoring, resp. changing of the temperature over the sound speed brings the advantage of the response rate to the change in the process. Temperature sensors have a certain delay that is caused by encapsulation of the sensor, in some cases from materials in which the temperature response is much slower. This can cause instability in the control process and to avoid it, the algorithmic processes in the control systems must have a certain delay. Temperature monitoring can be used in technological processes for different types of media. In view of the response rate mentioned above, it is possible to accelerate the process and thereby increase its efficiency.

Results of this study will be a part of next research in drinking water monitoring and analysis. The study will be provided as a technical concept for bacteria grow study in drinking water linings and supplying systems.

\section{Acknowledgments}

This paper was written in frame of the work for testing of ultrasonic systems in drinking water applications in company MEDON Austria in cooperation with Slovak University of Technology in Bratislava, UIBE, Trnava, Slovakia.

\section{References}

[1] Galea, S. C.; Chiu, W. K.; Nanasi, J. (1993). Journal of Intelligent Material Systems and Structures, 4.3: 330-336, Use of piezoelectric films in detecting and monitoring damage in composites.

[2] Gripp, Inc. (2018). Fluxus F601. Available from: https://grippinc.com/product/fluxus-f601/. Accessed: 2018-08-09

[3] Hayashi, Y.; Numata, K.; Nishizawa, K. (1994). The Journal of the Acoustical Society of America, U.S. Patent No $5,360,268$, Ultrasonic temperature measuring apparatus.

[4] https://electronics.stackexchange.com/questions/186449/what-is-the-fastest-temperature-sensor, (2018). Electrical engineering, Accessed: 2018-08-11 
[5] Králiková,R.; Sokolová, H. ; Wessely, E. \& Polak, J. (2013). Approaches to assessment of hot environment. DAAAM International Scientific Book, chapter 14. Vienna. DAAAM International. p. 317-328. ISBN 978-3901509-94-0

[6] Králiková,R.; Sokolová, H.\& Wessely, E. (2014). Thermal Environment Evaluation According to Indices in Industrial Workplaces. Procedia Engineering. No. 69, p. 158-167. ISSN $1877-$ 7058. DOI: $10.1016 /$ j.proeng.2014.02.216

[7] Králiková,R..\& Koblasa, F.(2018) Approaches to the evaluation of workshop microclimate conditions. MM Science Journal, p. 2397-2400. ISSN 1803-1269. DOI: 10.17973/MMSJ.2018_06_2017107

[8] Kraus, D. HSB. Labor für Technische Akustik. Temperaturabhängigkeit der Schallgeschwindigkeit in Flüssigkeiten. Available from: http://homepages.hs-bremen.de/ krausd/iwss/TA_V5c.pdf. Accessed: 2018-05-10

[9] Livengood, J. C.; Rona, Th P.; Baruch, J. J. (1954). The Journal of the Acoustical Society of America, 26.5: 824830, Ultrasonic temperature measurement in internal combustion engine chamber.

[10] Medon. Hülsenfühler/Kabeltemperaturfühler. Available from: https://medon.at/index.php/produkte/64-htf. Accessed: 2018-06-09

[11] National institute of standards and Technology. NIST Chemistry WebBook, SRD 69. Available from: https://webbook.nist.gov/cgi/fluid.cgi?P=1\&TLow=20\&THigh=70\&TInc=1\&Applet=on\&Digits=5\&ID=C773218 $5 \&$ Action=Load $\&$ Type $=I s o B a r \& T U n i t=C \& P U n i t=$ bar $\& D U n i t=k g \% 2 F m 3 \& H U n i t=k J \% 2 F m o l \& W U n i t=m \% 2 F s$ $\&$ VisUnit $=$ uPa*s\&STUnit=N\%2Fm\&RefState=DEF. Accessed: 2018-09-10

[12] National institute of standards and Technology. NIST Chemistry WebBook, SRD 69. Available from: https://webbook.nist.gov/cgi/fluid.cgi?P=1\&TLow=50\&THigh=95\&TInc=1\&Applet=on\&Digits=5\&ID=C773218 $5 \&$ Action=Load $\&$ Type $=I s o B a r \& T U n i t=C \& P U n i t=$ bar $\& D U n i t=k g \% 2 F m 3 \& H U n i t=\mathrm{kJ} \% 2 \mathrm{Fmol} \& W U n i t=\mathrm{m} \% 2 \mathrm{Fs}$ $\&$ VisUnit $=$ uPa*s\&STUnit=N\%2Fm\&RefState=DEF. Accessed: 2018-09-10

[13] LM34 Precision Fahrenheit Temperature Sensors. Available from: http://www.ti.com/lit/ds/symlink/lm34.pdf. Accessed: 2018-05-11

[14] Speed of sound in water. Available from: https://studopedia.su/17_47870_skorost-zvuka-v-vode.html. Accessed: 2018-08-15 